\title{
Cyclooxygenase- 2 contributes to the selective induction of cell death by the endocannabinoid 2-arachidonoyl glycerol in hepatic stellate cells
}

\author{
S.V. Siegmund ${ }^{\text {a, c, * }}$, A. Wojtalla ${ }^{\text {a, b, c, } 1}$, M. Schlosser ${ }^{\text {a, } 1}$, F.A. Schildberg ${ }^{\text {d }}$, P.A. Knolle ${ }^{\text {d }}$, \\ R.M. Nüsing ${ }^{\mathrm{e}}$, A. Zimmer ${ }^{\mathrm{b}}$, C.P. Strassburg ${ }^{\mathrm{a}}$, M.V. Singer ${ }^{\mathrm{c}}$ \\ ${ }^{a}$ Department of Internal Medicine I, University of Bonn, Germany \\ ${ }^{\mathrm{b}}$ Institute of Molecular Psychiatry, University of Bonn, Germany \\ ${ }^{\mathrm{c}}$ Department of Medicine II, University Hospital Mannheim, University of Heidelberg, Germany \\ ${ }^{\mathrm{d}}$ Institutes of Molecular Medicine and Experimental Immunology, University of Bonn, Germany \\ e Institute of Clinical Pharmacology, Johann Wolfgang Goethe-University Frankfurt, Germany
}

\section{A R T I C L E I N F O}

\section{Article history:}

Received 5 January 2016

Accepted 14 January 2016

Available online 20 January 2016

\section{Keywords:}

Cyclooxygenase-2

2-Arachidonoyl glycerol

Prostaglandin $\mathrm{D}_{2}$ glycerol ester

Hepatic stellate cells

Hepatocytes

Cell death

\begin{abstract}
A B S T R A C T
The endogenous cannabinoid 2-arachidonoyl glycerol (2-AG) is an anti-fibrotic lipid mediator that induces apoptosis in hepatic stellate cells (HSCs), but not in hepatocytes. However, the exact molecular mechanisms of this selective induction of HSC death are still unresolved. Interestingly, the inducible isoform of cyclooxygenase, COX-2, can metabolize 2-AG to pro-apoptotic prostaglandin glycerol esters (PG-GEs). We analyzed the roles of COX-2 and endocannabinoid-derived PG-GEs in the differential susceptibility of primary activated HSCs and hepatocytes toward 2-AG-induced cell death. HSCs displayed significant COX-2 expression in contrast to hepatocytes. Similar to 2-AG, treatment of HSCs with $\mathrm{PGD}_{2}$-GE dose-dependently induced cell death independently from cannabinoid receptors that was accompanied by PARP- and caspase 3-cleavage. In contrast to $2-\mathrm{AG}, \mathrm{PGD}_{2}-\mathrm{GE}$ failed to induce significant ROS formation in HSCs, and depletion of membrane cholesterol did not rescue HSCs from $\mathrm{PGD}_{2}$-GEinduced apoptosis. These findings indicate differential engagement of initial intracellular signaling pathways by 2-AG and its COX-2-derived metabolite $\mathrm{PGD}_{2}-\mathrm{GE}$, but similar final cell death pathways. Other PG-GEs, such as $\mathrm{PGE}_{2}$-or $\mathrm{PGF}_{2 \alpha}$-GE did not induce apoptosis in HSCs. Primary rat hepatocytes were mainly resistant against 2-AG- and $\mathrm{PGD}_{2}$-GE-induced apoptosis. $\mathrm{HSCs}$, but not hepatocytes were able to metabolize 2-AG to $\mathrm{PGD}_{2}-\mathrm{GE}$. As a proof of principle, HSCs from $\mathrm{COX}_{-2}{ }^{-1-}$ mice lacked $\mathrm{PDG}_{2}$-GE production after 2-AG treatment. Accordingly, COX-2 $2^{-1-}$ HSCs were resistant against 2-AG-induced apoptosis. In conclusion, the divergent expression of COX-2 in HSCS and hepatocytes contributes to the different susceptibility of these cell types towards 2-AG-induced cell death due to the generation of proapoptotic $\mathrm{PGD}_{2}$-GE by COX-2 in HSCs. Modulation of COX-2-driven metabolization of 2-AG may provide a novel physiological concept allowing the specific targeting of HSCs in liver fibrosis.
\end{abstract}

() 2016 Elsevier Inc. All rights reserved.

\section{Introduction}

The endocannabinoid system, consisting of arachidonic-acidderived lipid mediators, known as endocannabinoids, their specific receptors and enzymes for endocannabinoid biosynthesis and

\footnotetext{
* Corresponding author. Department of Internal Medicine I, University of Bonn, Sigmund-Freud-Str. 25, D-53105 Bonn, Germany.

E-mail address: soeren.siegmund@ukb.uni-bonn.de (S.V. Siegmund).

1 Equal contribution.
}

degradation, is critically implicated in the regulation of hepatic injury and fibrogenesis.

Although the endocannabinoid system is scarcely expressed in healthy liver, endocannabinoid receptors are upregulated and endocannabinoid levels increase significantly during diseased states of the organ [1-3]. Cannabinoid receptor $2^{-1-}$ mice displayed increased hepatic fibrogenesis in a model of $\mathrm{CCl}_{4}$-induced liver fibrosis, whereas $\mathrm{CB} 1^{-I-}$ mice showed reduced fibrogenesis $[4,5]$. However, the mechanisms by which the endocannabinoid system regulates liver injury and fibrogenesis are not entirely understood [3]. Endocannabinoids, such as AEA, 2-AG or N-arachidonoyl 
dopamine (NADA) display anti-fibrotic properties in the liver by selectively inducing cell death of activated hepatic stellate cells (HSCs), the main fibrogenic cell type in the liver, but not in hepatocytes $[1-3,6-8]$. Hepatocyte cell death is considered to promote fibrogenesis, whereas elimination of activated HSCs may represent a mechanism to attenuate hepatic fibrosis [9].

Endocannabinoids evoke a wide spectrum of physiological actions that are mostly effectuated through the G-protein coupled cannabinoid receptors CB1 and CB2 [10,11], but can also occur independently from these receptors $[1,2,6,12,13]$. Endocannabinoids were initially described in the central nervous system, where they are involved in the control of e.g. food intake, emotions, pain perception, or sleep [14]. Moreover, endocannabinoids have also been shown to regulate inflammation, cell proliferation, cell death and lipogenesis in peripheral organs such as the liver [15-19].

We previously demonstrated that 2-AG, the most abundant endocannabinoid in vertebrate animals, induced reactive oxygen species (ROS)-triggered, cannabinoid receptor-independent apoptosis in primary HSCs, but not in hepatocytes due to different intracellular levels of the antioxidant glutathione and unequal expression of the endocannabinoid-degrading enzyme fatty acid amide hydrolase (FAAH) in these cell types $[1,8]$. However, these factors alone do not fully explain the remarkable difference in susceptibility to 2-AG-mediated cell death. Since activated HSCs express significant amounts of the alternative 2-AGmetabolizing enzyme COX-2 [20] in contrast to hepatocytes [21], we sought to investigate, whether this discrepancy of COX-2 expression contributes to the differential susceptibility of 2-AGinduced apoptosis in these two hepatic cell populations.

\section{Materials and methods}

\subsection{Animals and primary cell isolation}

Primary HSCs were isolated from livers of balb/c wild-type (25-35 g, n = 30), C57BL/6J wild-type (25-35 g, n=4), COX-2 ${ }^{-l}$ $(25-35 \mathrm{~g}, \mathrm{n}=4)$ mice as previously described $[1,8]$. COX-2 ${ }^{-1-}$ mice [22] in a C57BL/6J background were kindly provided by $\mathrm{Dr}$. Rolf M. Nüsing (Johann Wolfgang Goethe-University, Frankfurt, Germany). Purity of HSC preparations was $94 \%$, as assessed by autofluorescence at day 2 after isolation. HSCs were cultured on uncoated plastic tissue culture dishes as described. HSCs were not passaged and considered culture-activated between day 7 and 14 after isolation. Primary hepatocytes were isolated from wild-type balb/c mice (25-35 g, $\mathrm{n}=6$ ) and cultured as described previously [2,7]. All animals received humane care and all procedures were approved by the local committees for animal studies (Regierungspräsidium Karlsruhe and LANUV Recklinghausen).

\subsection{Experimental model of liver fibrosis}

Liver fibrosis was induced in male balb/c wild-type mice (30-35 g, n = 4) in a model of cholestatic liver disease by ligating the common bile duct as described [1,7]. Sham-operated animals served as controls $(n=4)$. Seven or 14 days after surgery the mice were sacrificed and the livers were excised for protein and RNA isolation.

\subsection{Cell treatment and detection of cell death}

Hepatocytes were kept in serum-free HDM medium for $12 \mathrm{~h}$ before experiments. HSCs were serum-starved with serum-free DMEM for $12 \mathrm{~h}$. Cells were treated either with 2-AG, prostaglandin $D_{2}$, prostaglandin $D_{2}$ glycerol ester, prostaglandin $E_{2}$ glycerol ester, prostaglandin $\mathrm{F}_{2 \alpha}$ glycerol ester (all Cayman Chemical,
Ann Arbor, MI) or vehicle (ethanol; 0.1\% final concentration), or actinomycin D (Sigma-Aldrich, Deisenhofen, Germany) plus murine TNF $\alpha$ (R\&D Systems, Minneapolis, MN). Where indicated, cells were pretreated with the CB1 antagonist AM251 (Cayman Chemical), CB2 antagonist SR144528 (Sanofi-Aventis, Montpellier, France), pan-caspase inhibitor Z-VAD-FMK (R\&D Systems), membrane cholesterol depletor methyl- $\beta$-cyclodextrin, or the antioxidant Trolox (both Sigma-Aldrich). Cell death was measured by LDH release into the culture medium according to the manufacturer (Roche, Mannheim, Germany). Apoptosis was visualized by fluorescent microscopy using an annexin $\mathrm{V} /$ propidium iodide-staining kit (Roche).

\subsection{Measurement of prostaglandin glycerol ester levels}

The $\mathrm{PGD}_{2}$-GE concentration in lysates of primary mouse hepatocytes and HSCs was measured by a $\mathrm{PGD}_{2}$-MOX EIA kit according to the manufacturer's instructions (Cayman Chemical).

\subsection{Detection of ROS}

Reactive oxygen species formation was measured for the indicated time in a multiwell fluorescence plate reader (Fluostar Optima, BMG) with excitation and emission filters of $485 \mathrm{~nm}$ and $535 \mathrm{~nm}$, respectively, using the redox-sensitive dye 5-(and-6)chloromethyl-2', $7^{\prime}$-dichlorodihydrofluorescein diacetate (CM$\mathrm{H}_{2}$ DCFDA; Molecular Probes-Invitrogen, Darmstadt, Germany) as described $[1,8]$.

\subsection{Quantitative RT-PCR analysis}

RNA was isolated from serum-starved activated HSCs and hepatocytes and reverse transcribed as described $[1,2,8]$. Real time PCR was performed for 40 cycles of $15 \mathrm{~s}$ at $95^{\circ} \mathrm{C}$ and $60 \mathrm{~s}$ at $60{ }^{\circ} \mathrm{C}$ using an ABI 7900HT sequence detection system (Applied Biosystems, Darmstadt, Germany) as shown $[1,2,8]$, and subsequently analyzed with the gene expression assay for PTGS2 (Applied Biosystems; assay no. Mm00478374_m1).

\subsection{Flow cytometric analysis}

For intracellular staining of COX-2, cells were fixed with $2 \%$ paraformaldehyde in PBS with 1\% FBS/2 mM EDTA at RT. Afterward, cells were permeabilized with $0.5 \%$ saponin, followed by incubation with primary COX-2 antibody (Cayman Chemical) and Alexa647coupled secondary antibody on ice. The Fc gamma receptorblocking antibody (clone 2.4G2) was added to prevent nonspecific binding. Acquisition and analysis was conducted using a Canto II (BD Bioscience, Heidelberg, Germany) and FlowJo software (Tree Star Inc, Ashland, OR).

\subsection{Western blotting}

Electrophoresis of protein extracts and subsequent blotting were performed as described previously $[1,8]$. Blots were incubated with anti-COX-2, (Cayman Chemical); anti-caspase-3 or anti-poly(ADP-ribose) polymerase (anti-PARP, both Cell Signaling Technologies, Beverly, MA). Cytochrome c release was determined in S-100 fractions using anti-cytochrome c (BD PharMingen, San Jose, CA) as described [23]. Blots were reprobed with anti- $\beta$-actin antibody (Cell Signaling Technologies) as loading control.

\subsection{Statistical analysis}

All data represent the mean of three independent 
experiments \pm SEM. For the determination of statistical significance, unpaired Student's t-tests were performed using SigmaStat (SPSS, Chicago, IL). P values of $\leq 0.05$ were considered to be statistically significant.

\section{Results}

\subsection{Involvement of COX-2 in 2-AG-induced cell death in HSCS}

In parallel to the expression of the HSC activation marker $\alpha$-SMA COX-2 mRNA expression increased 54-fold during 14 days in BDLtreated livers (Fig. 1A, B). Interestingly, protein and mRNA levels of COX-2 in activated HSCs were 6,8 times higher compared to hepatocytes (Fig. 1C, D). It was recently shown that 2-AG is first metabolized by COX-2 to unstable $\mathrm{PGH}_{2}-\mathrm{GE}$. $\mathrm{PGH}_{2}-\mathrm{GE}$ acts as a substrate for PGD-, PGE- or PGF-synthases resulting in $\mathrm{PGD}_{2}-\mathrm{GE}$, $\mathrm{PGE}_{2}-\mathrm{GE}$ or $\mathrm{PGF}_{2 \alpha}-\mathrm{GE}$, respectively [24]. To check whether these potential metabolites of 2-AG were involved in 2-AG-induced cell death, and to exclude that prostaglandin $\mathrm{D}_{2}\left(\mathrm{PGD}_{2}\right)$, the COX-2 metabolite of arachidonic acid, may also contribute to 2-AGmediated cell death, primary HSCs were exposed to $100 \mu \mathrm{M}$ of each substance. Interestingly, only $\mathrm{PGD}_{2}-\mathrm{GE}$ induced cell death in primary activated HSCs (Fig. 1E). We were further interested in a possible differential metabolization of $2-\mathrm{AG}$ to $\mathrm{PGD}_{2}-\mathrm{GE}$ by
A

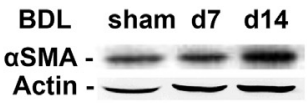

C

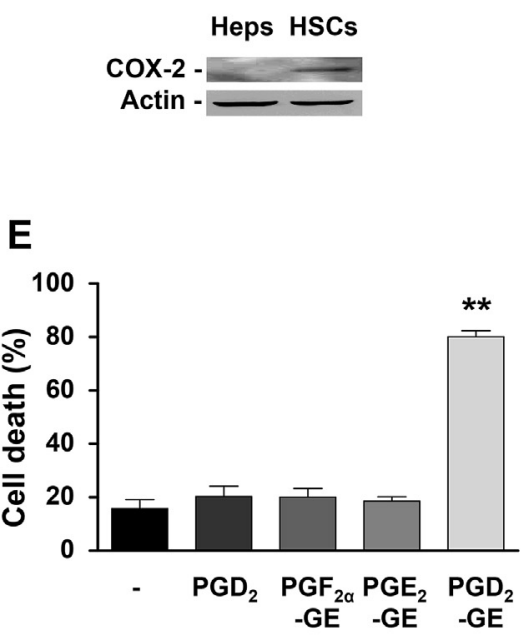

G

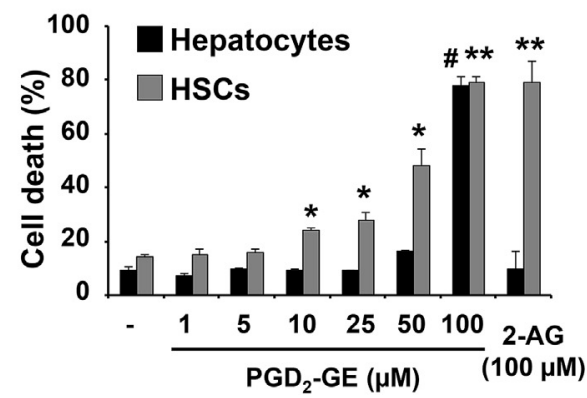

B
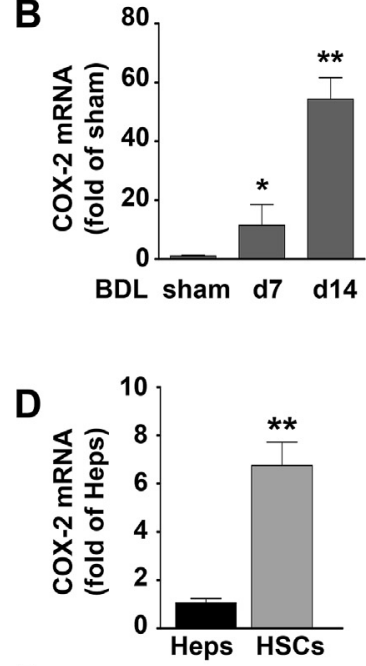

$\mathbf{F}$

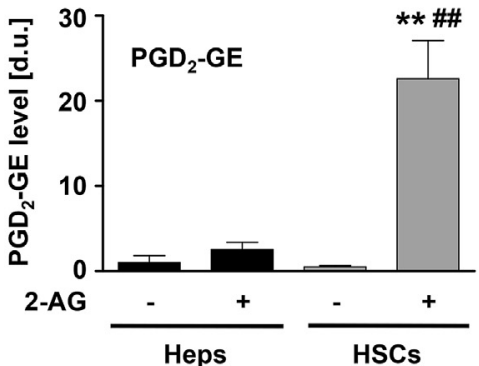

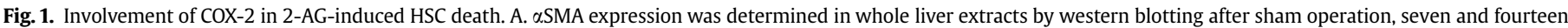

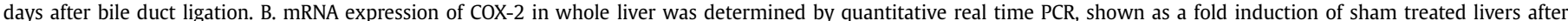

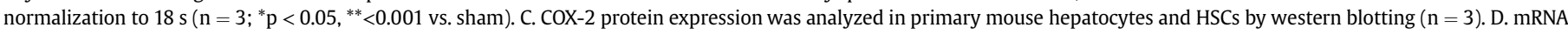

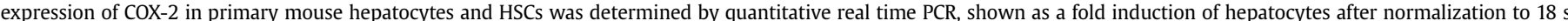

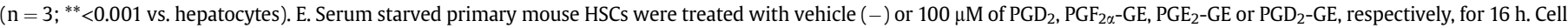

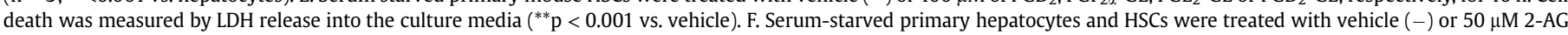

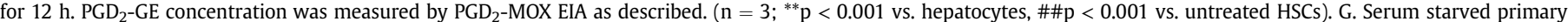

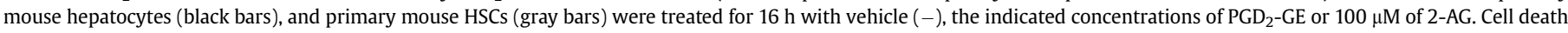
was determined by LDH release $\left(\mathrm{n}=3 ;{ }^{*} \mathrm{p}<0.05,{ }^{* *} \mathrm{p}<0.001\right.$ vs. vehicle treated HSCs, $\# \mathrm{p}<0.05$ vs. vehicle treated hepatocytes). 

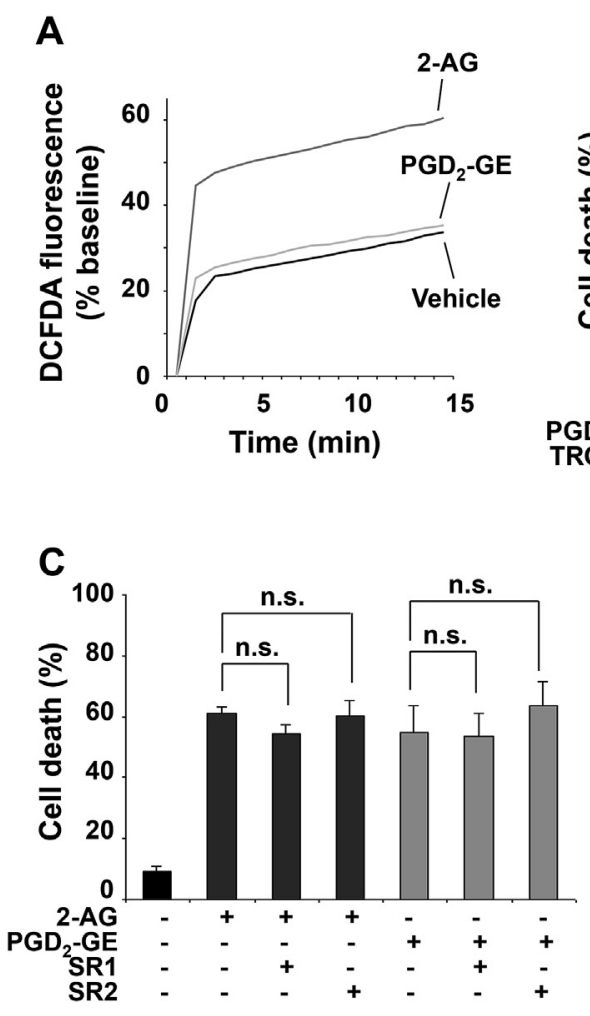

\section{E}

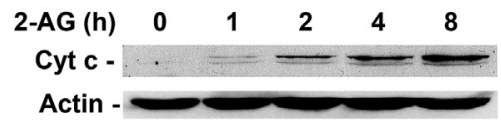

$\mathbf{F}$

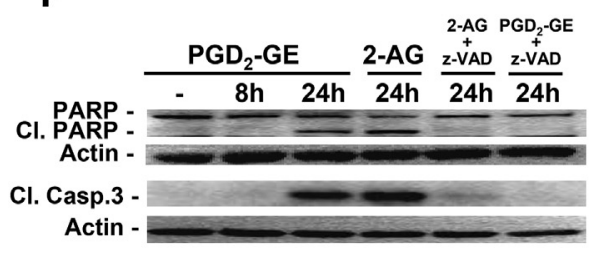

B

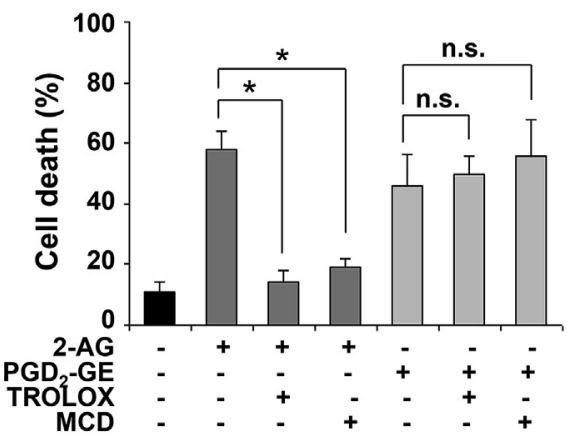

D

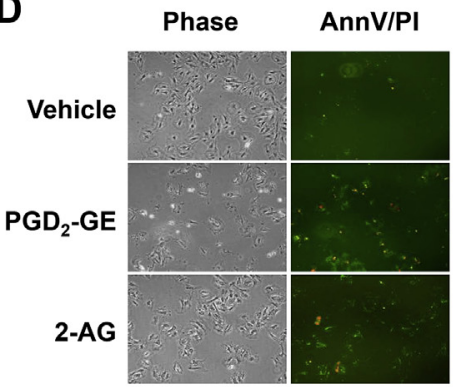

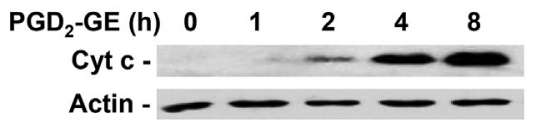

$\mathbf{G}$

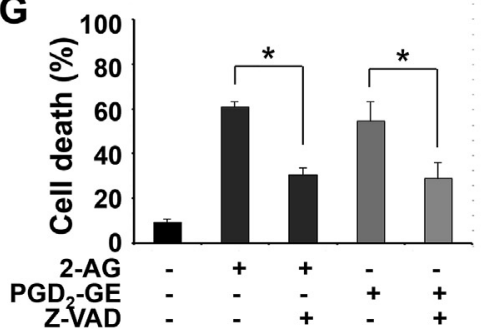

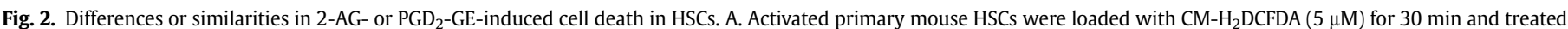

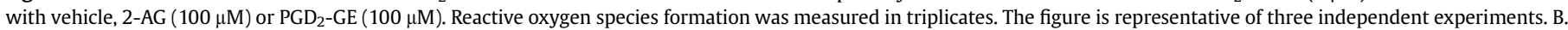

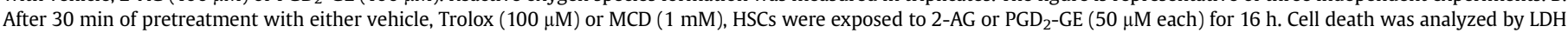

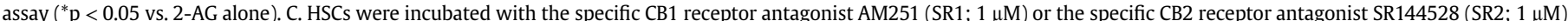

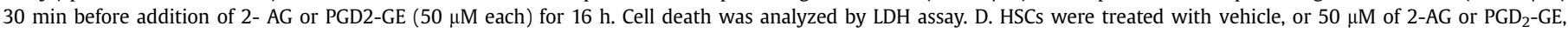

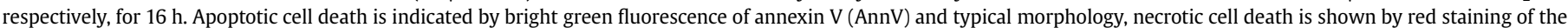

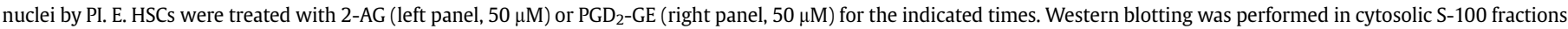

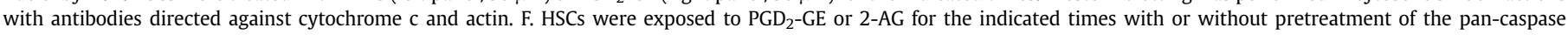

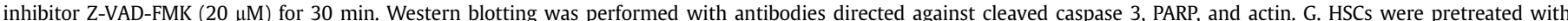

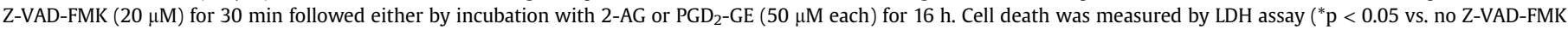
treatment). (For interpretation of the references to colour in this figure legend, the reader is referred to the web version of this article.)

hepatocytes or HSCs. After stimulation of these cell types with 2-AG or vehicle for $16 \mathrm{~h}$ (Fig. $1 \mathrm{~F}$ ), no difference in $\mathrm{PGD}_{2}$-GE levels was observed between vehicle-treated hepatocytes and HSCs as well as vehicle-treated and 2-AG-stimulated hepatocytes. However, there was a significant induction of $\mathrm{PGD}_{2}$-GE production in 2-AG-stimulated HSCs reaching a 22-fold higher $\mathrm{PGD}_{2}-\mathrm{GE}$ level compared to vehicle-treated $\mathrm{HSCs}$ and a 15 -fold higher $\mathrm{PGD}_{2}$-GE level compared to 2-AG-treated hepatocytes. When we exposed hepatocytes and HSCs to increasing concentrations of $\mathrm{PGD}_{2}-\mathrm{GE}$, HSCs showed a significantly higher susceptibility toward $\mathrm{PGD}_{2}$-GE-induced cell death than hepatocytes. Hepatocytes underwent cell death only after exposure to a excessive $\mathrm{PGD}_{2}-\mathrm{GE}$ concentration of $100 \mu \mathrm{M}$, but not after 2-AG in the same concentration. However, since hepatocytes do not significantly express COX-2, it is unlikely that intracellular concentrations of $\mathrm{PGD}_{2}-\mathrm{GE}$ reach substantial cytotoxic levels in this cell type. 
A

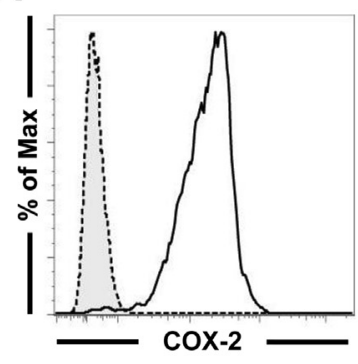

B

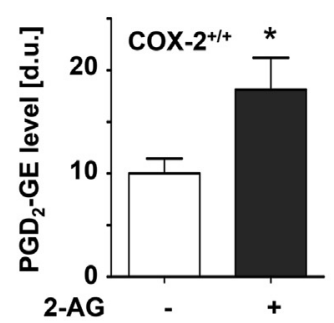

C

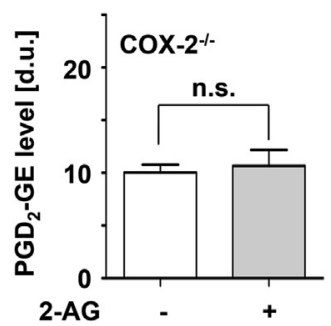

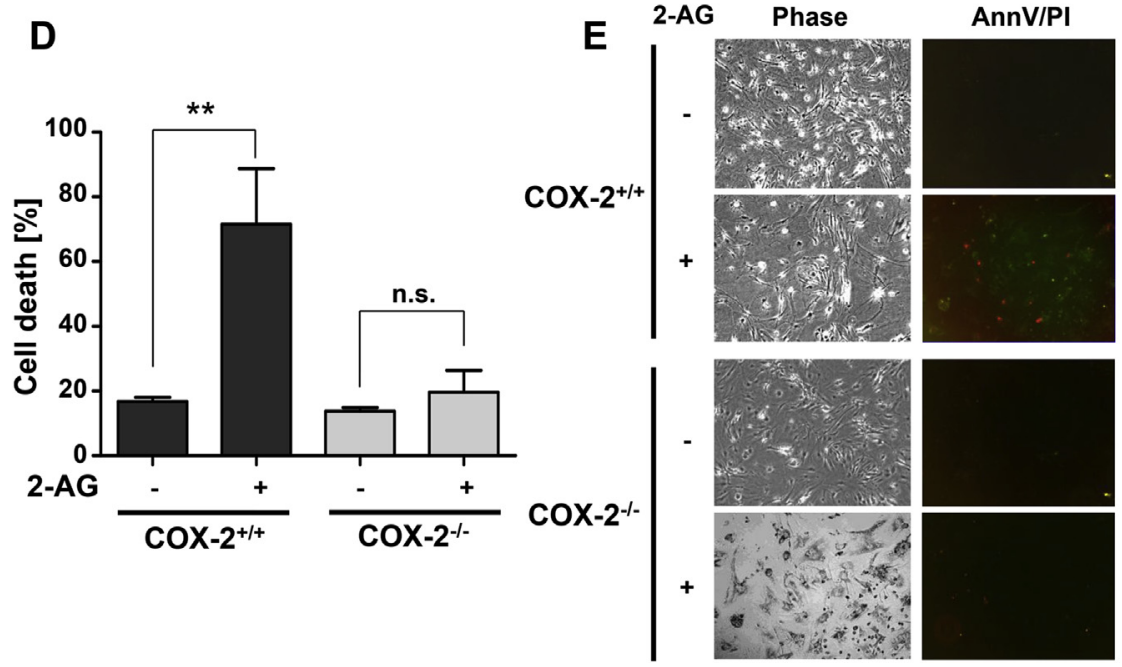

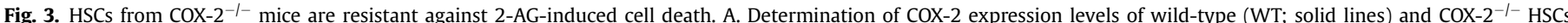

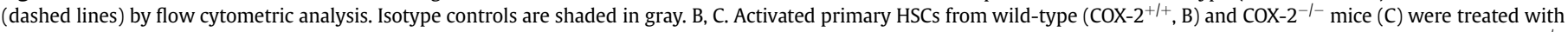

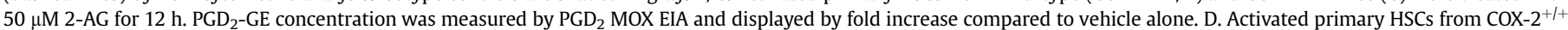

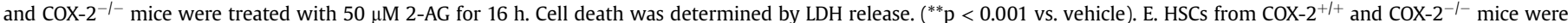

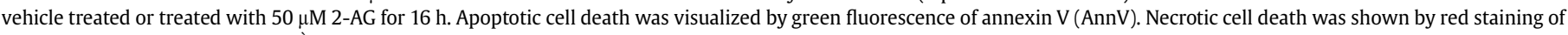
the nuclei by propidium iodide (PI). (For interpretation of the references to colour in this figure legend, the reader is referred to the web version of this article.)

\subsection{Comparison between mechanisms of 2-AG- and $P G D_{2}-G E-$ induced cell death in HSCs}

Next we investigated whether $\mathrm{PGD}_{2}-\mathrm{GE}$ accounts for key features of 2-AG-induced HSC cell death. $\mathrm{PGD}_{2}$-GE did not cause significant ROS production in HSCs in contrast to 2-AG (Fig. 2A). Accordingly, antioxidant pretreatment of HSCs by Trolox ameliorated 2-AG-, but not $\mathrm{PGD}_{2}-\mathrm{GE}-$ induced cell death (Fig. $2 \mathrm{~B}$ ). Moreover, in a previous study we demonstrated that 2-AG required membrane cholesterol interaction in cholesterol-rich lipid rafts to cause cell death in HSCs [1]. PGD $_{2}$-GE-induced HSC death on the contrary could not be blocked by prior membrane cholesterol depletion by MCD (Fig. 2B). Pretreatment of HSCs with the specific CB1 or CB2 receptor antagonists AM251 or SR144528, respectively, did neither prevent 2-AG- nor $\mathrm{PGD}_{2}-\mathrm{GE}$-mediated cell death (Fig. 2C). These data suggest, that $\mathrm{PGD}_{2}-\mathrm{GE}$ does not require interaction with membrane cholesterol or cannabinoid receptors for cellular uptake and subsequent induction of cell death in HSCs since it is produced intracellularly by COX-2 from 2-AG. We next compared the mode of cell death caused by 2-AG and its metabolite $\mathrm{PGD}_{2}-\mathrm{GE}$. As a common final pathway, both substances induced distinct positive Annexin V-staining in HSCs indicating apoptotic cell death (Fig. 2D). Apoptotic cell death caused by 2-AG as well as $\mathrm{PGD}_{2}-\mathrm{GE}$ was preceded by an increase in cytochrome $\mathrm{c}$ in $\mathrm{S} 100$ fractions starting after $2 \mathrm{~h}$ of treatment (Fig. 2E, left and right panel). Cytochrome $\mathrm{c}$ is released from mitochondria undergoing mitochondrial permeability transition and triggers activation of executioner caspases, such as caspase 3 [23]. Accordingly, HSC treatment with $25 \mu \mathrm{M}$ of $2-\mathrm{AG}$ or $\mathrm{PGD}_{2}-\mathrm{GE}$, respectively, led to caspase 3- and PARP-cleavage, two hallmarks of apoptotic cell death (Fig. 2F). Interestingly, caspase 3 and PARP-cleavage could be blocked both in 2-AG- and $\mathrm{PGD}_{2}$-GE-mediated apoptosis by the pan-caspase inhibitor Z-VAD-FMK (Fig. 2F). Moreover, inhibition of caspase activation by Z-VAD-FMK significantly reduced 2-AG- as well as $\mathrm{PGD}_{2}$-GE-induced HSC apoptosis (Fig. 2G).

\subsection{Genetic inactivation of COX-2 renders HSCs resistant against 2 - AG-induced cell death}

To prove the importance of COX-2 in the mechanisms involved in 2-AG-mediated cell death in HSCs, we sought to apply HSCs isolated from $\mathrm{COX}-2^{-1-}$ mice and to compare these to wild-type HSCs. First, we confirmed, that activated HSCs isolated from COX2 wild-type mice indeed expressed significant amounts of COX-2 protein, whereas $\mathrm{COX}-2^{-1-}$ HSCs lacked the enzyme completely (Fig. 3A). We then exposed activated wild-type and COX-2-l- HSCs to 2-AG and measured the levels of $\mathrm{PGD}_{2}-\mathrm{GE}$ production (Fig. $3 \mathrm{~B}, \mathrm{C}$ ). In contrast to wild-type HSCs, COX-2 $2^{-1-}$ HSCs did not produce noticeable amounts of $\mathrm{PGD}_{2}$-GE (Fig. 3C). Thus, COX-2-l- HSCs were resistant against 2-AG-induced cell death in contrast to their wild-type counterparts (Fig. 3D). We further visualized the remarkable difference in cell death susceptibility of wild-type and 
COX-2 $2^{-l-}$ HSCs with Annexin V/PI staining (Fig. 3E).

\section{Discussion}

Although liver fibrosis has been regarded as irreversible for a long time, recent studies in animal models and patients have suggested that hepatic fibrosis is, at least to some degree, a reversible process [9]. Cell death of activated HSCs as the key fibrogenic cell population has been linked to the reversal of liver fibrosis. Previously, we and others demonstrated, that endocannabinoids are able to selectively induce cell death in activated HSCs, but not in hepatocytes $[1,2,4,6-8]$ and therefore exhibit antifibrogenic properties. However, the mechanisms by which endocannabinoids regulate liver injury and fibrogenesis are not completely understood [3]. Cell death in activated HSCs by endocannabinoids occurs independently from cannabinoid receptors through interaction with cholesterol-rich lipid rafts in the cell membrane $[1,2,6]$. In this study we show that 2-AG, the most abundant endocannabinoid in vertebrates, induces a burst of mitochondrial-derived ROS in HSCs, leading to cytochrome $\mathrm{C}$ release and subsequent activation of effector caspases such as caspase 3, resulting in apoptotic cell death. Divergence in cell death susceptibility in HSCs and hepatocytes are based on differences in the capability to terminate 2-AGmediated intracellular events: (i) hepatocytes possess higher levels of antioxidant glutathione to mitigate ROS production and (ii) significantly express the endocannabinoid-hydrolyzing enzyme FAAH $[1,8]$. Thus, the metabolization of endocannabinoids plays a pivotal role in the control of endocannabinoid signaling. Interestingly, besides the hydrolytic degradation pathways of 2-AG by FAAH or monoacyl glycerol lipase (MGL), the molecule may also be metabolized by oxygenases due to their high affinity to the arachidonyl moiety of 2-AG. In contrast to AEA, the oxygenase COX-2 specifically metabolizes 2-AG as efficiently as arachidonic acid [24]. Contrarily, the constitutionally active, non-inducible isoform COX-1 is not able to metabolize 2-AG [24]. Here we demonstrate that the expression of $\mathrm{COX}-2$ increased 54 -fold during hepatic fibrogenesis and activated HSCs largely contributed to this increase of COX-2 expression. Upregulation of COX-2 expression during fibrogenesis correlates with increased expression of the HSC activation marker $\alpha$-SMA (Fig. 1A) and rising hepatic levels of 2-AG [1]. In contrast to hepatocytes, activated HSCs were able to considerably metabolize 2-AG to $\mathrm{PGD}_{2}-\mathrm{GE}$, which represents a biologically highly active prostaglandin glycerol ester in endocannabinoid signaling [25]. $\mathrm{PGD}_{2}-\mathrm{GE}$ appears to be produced intracellularly, since it did not signal through $\mathrm{CB} 1$ - or $\mathrm{CB} 2$-receptors and, in contrast to 2-AG, did not interact with membrane cholesterol. Although $\mathrm{PGD}_{2}$-GE did not generate mitochondrial ROS, it induced the same final death signaling pathway including cytochrome $\mathrm{C}$ release and activation of executioner caspases like its parental substance 2-AG (see the graphical abstract). The importance of COX-2 as an endocannabinoid-degrading enzyme has been demonstrated, if other degradation enzymes, i.e. FAAH or MGL, are lacking [26]. We have previously shown, that HSCs do not express FAAH, but MGL $[7,8]$. However, expression of MGL does not rescue HSCs from 2-AG-induced cell death [8]. MGL may rather play a role in terminating $\mathrm{PGD}_{2}$-GE signaling through hydrolyzation [27]. MGL becomes downregulated in the liver during fibrogenesis in contrast to COX-2 $[8,28]$, which may in turn contribute to an increase in $\mathrm{PGD}_{2}$-GE production and thus an increase in 2-AG-induced death of HSCs. Other inflammatory cells in the liver, e.g. Kupffer cells also express high amounts of COX-2, but also FAAH and MGL (data not shown) as well as significant levels of GSH [29]. Therefore, similar to hepatocytes, Kupffer cells seem to be protected $a$ priori against 2AG-induced cell death.

Finally, the high resistance of $\mathrm{COX}-2^{-1-}$ HSCs against 2-AG- induced cell death confirms the importance of COX-2 in the differential susceptibility of HSCs and hepatocytes towards endocannabinoid-mediated toxicity.

In conclusion, 2-AG selectively induces cell death in activated HSCs due to (i) low levels of glutathione, (ii) low expression of degradation enzymes such as FAAH and (iii) high levels of COX-2, leading to production of pro-apoptotic $\mathrm{PGD}_{2}-\mathrm{GE}$. Consequently, modulation of COX-2-driven metabolization of 2-AG may provide a novel physiological concept allowing the specific targeting of HSCs in liver fibrosis.

\section{Acknowledgments}

The study was supported by Deutsche Forschungsgemeinschaft Grants SI 1366/1-1 and SFB TRR57 Project 15 (to SVS), SFB TRR57 Project 11 (to PAK) and FOR926 and SFB TRR57 Project 15 (to AZ).

\section{Transparency document}

Transparency document related to this article can be found online at http://dx.doi.org/10.1016/j.bbrc.2016.01.083.

\section{References}

[1] S.V. Siegmund, T. Oian, S. de Minicis, J. Harvey-White, G. Kunos, K.Y. Vinod, B. Hungund, R.F. Schwabe, The endocannabinoid 2-arachidonoyl glycerol induces death of hepatic stellate cells via mitochondrial reactive oxygen species, Faseb J. 21 (2007) 2798-2806.

[2] A. Wojtalla, F. Herweck, M. Granzow, S. Klein, J. Trebicka, S. Huss, R. Lerner B. Lutz, F.A. Schildberg, P.A. Knolle, T. Sauerbruch, M.V. Singer, A. Zimmer, S.V. Siegmund, The endocannabinoid N-arachidonoyl dopamine (NADA) selectively induces oxidative stress-mediated cell death in hepatic stellate cells, but not in hepatocytes, Am. J. Physiol. Gastrointest. Liver Physiol. 302 (2012) G873-G887.

[3] S.V. Siegmund, R.F. Schwabe, Endocannabinoids and liver disease. II. Endocannabinoids in the pathogenesis and treatment of liver fibrosis, Am. J. Physiol. Gastrointest. Liver Physiol. 294 (2008) G357-G362.

[4] B. Julien, P. Grenard, F. Teixeira-Clerc, J.T. Van Nhieu, L. Li, M. Karsak, A. Zimmer, A. Mallat, S. Lotersztajn, Antifibrogenic role of the cannabinoid receptor CB2 in the liver, Gastroenterology 128 (2005) 742-755.

[5] F. Teixeira-Clerc, B. Julien, P. Grenard, J. Tran Van Nhieu, V. Deveaux, L. Li, V. Serriere-Lanneau, C. Ledent, A. Mallat, S. Lotersztajn, CB1 cannabinoid receptor antagonism: a new strategy for the treatment of liver fibrosis, Nat. Med. 12 (2006) 671-676.

[6] S.V. Siegmund, H. Uchinami, Y. Osawa, D.A. Brenner, R.F. Schwabe, Anandamide induces necrosis in primary hepatic stellate cells, Hepatology 41 (2005) 1085-1095.

[7] S.V. Siegmund, E. Seki, Y. Osawa, H. Uchinami, B.F. Cravatt, R.F. Schwabe, Fatty acid amide hydrolase determines anandamide-induced cell death in the liver, J. Biol. Chem. 281 (2006) 10431-10438.

[8] S.V. Siegmund, A. Wojtalla, M. Schlosser, A. Zimmer, M.V. Singer, Fatty acid amide hydrolase but not monoacyl glycerol lipase controls cell death induced by the endocannabinoid 2-arachidonoyl glycerol in hepatic cell populations, Biochem. Biophys. Res. Commun. 437 (2013) 48-54.

[9] C. Trautwein, S.L. Friedman, D. Schuppan, M. Pinzani, Hepatic fibrosis: concept to treatment, J. Hepatol. 62 (2015) S15-S24.

[10] W.A. Devane, L. Hanus, A. Breuer, R.G. Pertwee, L.A. Stevenson, G. Griffin, D. Gibson, A. Mandelbaum, A. Etinger, R. Mechoulam, Isolation and structure of a brain constituent that binds to the cannabinoid receptor, Science 258 (1992) 1946-1949.

[11] S. Munro, K.L. Thomas, M. Abu-Shaar, Molecular characterization of a peripheral receptor for cannabinoids, Nature 365 (1993) 61-65.

[12] M. Begg, P. Pacher, S. Batkai, D. Osei-Hyiaman, L. Offertaler, F.M. Mo, J. Liu, G. Kunos, Evidence for novel cannabinoid receptors, Pharmacol. Ther. 106 (2005) 133-145.

[13] C.E. Rockwell, N.T. Snider, J.T. Thompson, J.P. Vanden Heuvel, N.E. Kaminski, Interleukin-2 suppression by 2-arachidonyl glycerol is mediated through peroxisome proliferator-activated receptor gamma independently of cannabinoid receptors 1 and 2, Mol. Pharmacol. 70 (2006) 101-111.

[14] V. Di Marzo, M. Bifulco, L. De Petrocellis, The endocannabinoid system and its therapeutic exploitation, Nat. Rev. Drug Discov. 3 (2004) 771-784.

[15] T.W. Klein, Cannabinoid-based drugs as anti-inflammatory therapeutics, Nat. Rev. Immunol. 5 (2005) 400-411.

[16] M. Maccarrone, A. Finazzi-Agro, The endocannabinoid system, anandamide and the regulation of mammalian cell apoptosis, Cell Death Differ. 10 (2003) 946-955.

[17] D. Osei-Hyiaman, M. DePetrillo, P. Pacher, J. Liu, S. Radaeva, S. Batkai, 
J. Harvey-White, K. Mackie, L. Offertaler, L. Wang, G. Kunos, Endocannabinoid activation at hepatic CB1 receptors stimulates fatty acid synthesis and contributes to diet-induced obesity, J. Clin. Invest 115 (2005) 1298-1305.

[18] W.I. Jeong, D. Osei-Hyiaman, O. Park, J. Liu, S. Batkai, P. Mukhopadhyay, N. Horiguchi, J. Harvey-White, G. Marsicano, B. Lutz, B. Gao, G. Kunos, Paracrine activation of hepatic CB1 receptors by stellate cell-derived endocannabinoids mediates alcoholic fatty liver, Cell Metab. 7 (2008) 227-235.

[19] M. Maccarrone, I. Bab, T. Biro, G.A. Cabral, S.K. Dey, V. Di Marzo, J.C. Konje, G. Kunos, R. Mechoulam, P. Pacher, K.A. Sharkey, A. Zimmer, Endocannabinoid signaling at the periphery: 50 years after THC, Trends Pharmacol. Sci. 36 (2015) 277-296.

[20] Y.H. Paik, J.K. Kim, J.I. Lee, S.H. Kang, D.Y. Kim, S.H. An, S.J. Lee, D.K. Lee K.H. Han, C.Y. Chon, S.I. Lee, K.S. Lee, D.A. Brenner, Celecoxib induces hepatic stellate cell apoptosis through inhibition of Akt activation and suppresses hepatic fibrosis in rats, Gut 58 (2009) 1517-1527.

[21] P. Martin-Sanz, R. Mayoral, M. Casado, L. Bosca, COX-2 in liver, from regeneration to hepatocarcinogenesis: what we have learned from animal models? World J. Gastroenterol. 16 (2010) 1430-1435.

[22] S.G. Morham, R. Langenbach, C.D. Loftin, H.F. Tiano, N. Vouloumanos, J.C. Jennette, J.F. Mahler, K.D. Kluckman, A. Ledford, C.A. Lee, O. Smithies, Prostaglandin synthase 2 gene disruption causes severe renal pathology in the mouse, Cell 83 (1995) 473-482.

[23] R.F. Schwabe, H. Uchinami, T. Qian, B.L. Bennett, J.J. Lemasters, D.A. Brenner,
Differential requirement for c-Jun NH2-terminal kinase in TNFalpha- and Fasmediated apoptosis in hepatocytes, Faseb J. 18 (2004) 720-722.

[24] K.R. Kozak, L.J. Marnett, Oxidative metabolism of endocannabinoids, Prostagl. Leukot. Essent. Fat. Acids 66 (2002) 211-220.

25] M. Alhouayek, J. Masquelier, P.D. Cani, D.M. Lambert, G.G. Muccioli, Implication of the anti-inflammatory bioactive lipid prostaglandin D2-glycerol ester in the control of macrophage activation and inflammation by ABHD6, Proc Natl. Acad. Sci. U. S. A. 110 (2013) 17558-17563.

[26] A. Weber, J. Ni, K.H. Ling, A. Acheampong, D.D. Tang-Liu, R. Burk, B.F. Cravatt, D. Woodward, Formation of prostamides from anandamide in FAAH knockout mice analyzed by HPLC with tandem mass spectrometry, J. Lipid Res. 45 (2004) 757-763.

[27] J.R. Savinainen, E. Kansanen, T, Pantsar, D. Navia-Paldanius, T, Parkkari, M. Lehtonen, T. Laitinen, T. Nevalainen, A. Poso, A.L. Levonen, J.T. Laitinen, Robust hydrolysis of prostaglandin glycerol esters by human monoacylglycerol lipase (MAGL), Mol. Pharmacol. 86 (2014) 522-535.

[28] P. Mai, L. Yang, L. Tian, L. Wang, S. Jia, Y. Zhang, X. Liu, L. Yang, L. Li, Endocannabinoid system contributes to liver injury and inflammation by activation of bone marrow-derived monocytes/macrophages in a CB1-dependent manner, J. Immunol. 195 (2015) 3390-3401.

[29] S.W. Luckey, D.R. Petersen, Activation of Kupffer cells during the course of carbon tetrachloride-induced liver injury and fibrosis in rats, Exp. Mol. Pathol. 71 (2001) 226-240. 